\title{
Escala para Avaliação de Náuseas e Vômitos Relacionados à Quimioterapia: Tradução e Adaptação Transcultural
}

doi: https://doi.org/10.32635/2176-9745.RBC.2022v68n1.1423

\author{
Scale for the Assessment of Chemotherpy-Induced Nausea and Vomits: Translation and Transcultural Adaptation \\ Escala de Evaluación de Náuseas y Vómitos Relacionados con la Quimioterapia: Traducción y Adaptación Cultural
}

Geovanna Maria Isidoro'; Ana Cristina Gonçalves Ferreira²; Eliza Mara das Chagas Paiva ${ }^{3}$; Jodi Dee Hunt Ferreira do Amaral4; Everson Cristiano de Abreu Meireles ${ }^{5}$; Ana Cláudia Mesquita Garcia ${ }^{6}$

RESUMO

Introduçáo: Náuseas e vômitos induzidos por quimioterapia acometem cerca de 70-80\% dos pacientes com câncer. Assim, é importante a utilização de um instrumento para avaliar melhor esses sintomas, visando a um tratamento mais adequado. Objetivo: Traduzir e adaptar culturalmente a escala Morrow Assessment of Nausea and Emesis para o contexto brasileiro. Método: Estudo correlacional do tipo survey, com tradução e adaptação cultural da escala segundo o protocolo da European Organization for Research and Treatment of Cancer - Quality of Life Group (EORTC-QLG). A amostra foi constituída por 160 pacientes em tratamento quimioterápico em uma clínica de oncologia. No processo de validação, realizaram-se análises de correlação multimétodos entre os itens da escala Morrow Assessment of Nausea and Emesis e os escores das escalas visuais numéricas de náusea e vômito com nível de $\mathrm{p}<0,05$. Resultados: $\mathrm{O}$ autor da escala autorizou a tradução. A escala Morrow Assessment of Nausea and Emesis e as escalas numéricas apresentaram correlaçóes significativas $(\mathrm{p}<0,01 ; \mathrm{p}<0,05)$, sendo que os itens que apresentaram correlação mais forte das escalas numéricas foram os que se referiram à avaliação de náusea e vômito pós-quimioterapia. Já os itens destinados à avaliação desses sintomas no momento pré-quimioterapia e ao uso da medicação antiemética e sua eficácia apresentaram associaçóes fracas com as escalas numéricas. Conclusáo: A escala Morrow Assessment of Nausea and Emesis apresentou-se adequada para a avaliação de náuseas e vômitos induzidos por quimioterapia no contexto brasileiro.

Palavras-chave: estudo de validação; náusea; vômito; tratamento farmacológico; oncologia.

\begin{abstract}
Introduction: Chemotherapy-induced nausea and vomiting affects nearly $70-80 \%$ of patients with cancer. To achieve a better treatment it is important to utilize an adequate instrument to assess these symptoms. Objective: To translate and culturally adapt the Morrow Assessment of Nausea and Emesis Scale to the Brazilian context. Method: Survey and correlational study, with the translation and cultural adaptation of the scale according to the protocol of the European Organization for Research and Treatment of Cancer - Quality of Life Group (EORTC-QLG). The sample consisted of 160 patients undergoing chemotherapy treatment in an oncology clinic. In the validation process, multimethod correlation analyses were carried out among the items of the Morrow Assessment of Nausea and Emesis Scale items and the scores of the numerical visual scales of nausea and vomits at the level of $\mathrm{p}<0.05$. Results: The author of the scale approved the translation process. The Morrow Assessment of Nausea and Emesis scale and the numerical scales presented significant correlations $(\mathrm{p}<0.01 ; \mathrm{p}<0.05)$, considering that the items presenting stronger correlation with the numerical scales were those addressing post-chemotherapy assessment of nausea and vomit. On the other hand, the items for pre-chemotherapy assessment of these symptoms and use of the antiemetic drugs and their efficacy presented weak associations with the numerical scales. Conclusion: The Morrow Assessment of Nausea and Emesis scale was adequate for the assessment of chemotherapy-induced nausea and vomiting in the Brazilian context. Key words: validation study; nausea; vomiting; drug therapy; medical oncology.
\end{abstract}

\section{RESUMEN}

Introducción: Las náuseas y vómitos inducidos por la quimioterapia afectan aproximadamente al 70-80\% de los pacientes con cáncer. Por lo tanto, es importante utilizar un instrumento para evaluar mejor estos síntomas, con el objetivo de un tratamiento más adecuado. Objetivo: Traducir y adaptar culturalmente la escala de Morrow Assessment of Nausea and Emesis al contexto brasileño. Método: Estudio correlativo del tipo de encuesta, con la traducción y adaptación cultural de la escala según el protocolo de la European Organization for Research and Treatment of Cancer - Quality of Life Group (EORTC-QLG). La muestra consistió en 160 pacientes sometidos a quimioterapia en una clínica oncológica. En el proceso de validación, se realizaron análisis de correlación multimétodos entre los elementos de la escala de Morrow Assessment of Nausea and Emesis y las puntuaciones de las escalas visuales numéricas de náuseas y vómitos con nivel de $\mathrm{p}<0,05$. Resultados: El autor de la escala autorizó la traducción. La Morrow Assessment of Nausea and Emesis y las escalas numéricas mostraron correlaciones significativas $(\mathrm{p}<0,01 ; \mathrm{p}<0,05)$, y los elementos que presentaron una correlación más fuerte de las escalas numéricas fueron los que se refirieron a la evaluación de las náuseas y los vómitos después de la quimioterapia. Por otro lado, los elementos destinados a la evaluación de estos síntomas en el momento anterior a la quimioterapia y el uso de medicamentos antieméticos y su eficacia presentaron asociaciones débiles con escalas numéricas. Conclusión: La Morrow Assessment of Nausea and Emesis fue adecuada para la evaluación de náuseas y vómitos inducidos por quimioterapia en el contexto brasileño.

Palabras clave: estudio de validación; náusea; vómitos; quimioterapia; oncología médica.

\footnotetext{
1,2,3,6Universidade Federal de Alfenas. Escola de Enfermagem. Alfenas (MG), Brasil. E-mails: geosergio01@gmail.com; ana_gferreira@hotmail.com; elizamdcp@gmail.com; ana. mesquita@unifal-mg.edu.br. Orcid iD: https://orcid.org/0000-0001-9847-6645; Orcid iD: https://orcid.org/0000-0003-3753-0075; Orcid iD: https://orcid.org/00000003-3489-8536; Orcid iD: https://orcid.org/0000-0001-9793-7905

${ }^{4}$ Universidade Federal de Uberlândia. Gerência de Psicologia e Psicopedagogia da Saúde do Hospital das Clínicas. Uberlândia (MG), Brasil. E-mail: jdhfda@gmail.com. Orcid iD: https://orcid.org/0000-0003-1534-129X

${ }^{5}$ Universidade Federal do Recôncavo da Bahia. Centro de Ciências da Saúde. Santo Antônio de Jesus (BA), Brasil. E-mail: eversoncam@yahoo.com.br. Orcid iD: https://orcid.org/0000-0002-1715-006X

Endereço para correspondência: Ana Cláudia Mesquita Garcia. Rua Gabriel Monteiro da Silva, 700 - Centro. Alfenas (MG), Brasil. CEP 37130-001.

E-mail: ana.mesquita@unifal-mg.edu.br
} 


\section{INTRODUÇÃO}

O câncer é considerado um importante problema de saúde pública ao redor do mundo, sendo responsável por cerca de $30 \%$ de mortes prematuras por doenças não transmissíveis entre adultos de 30 a 69 anos de idade ${ }^{1}$. Até o ano de 2040, 37 milhóes de pessoas no mundo serão acometidas pela doença ${ }^{1}$. No Brasil, maior país da América do Sul, são esperados 625 mil casos novos para cada ano do triênio 2020-2022 2 .

A quimioterapia antineoplásica é um dos principais tipos de tratamento para as doenças oncológicas. No entanto, em razáo da baixa ou nenhuma seletividade às células tumorais, esses fármacos podem levar ao surgimento de efeitos colaterais angustiantes, como náuseas e vômitos, os quais acometem cerca de $70-80 \%$ dos pacientes com câncer ${ }^{3,4}$.

Náuseas e vômitos induzidos por quimioterapia (NVIQ) são responsáveis por diminuir notavelmente a qualidade de vida desses pacientes, visto que podem afetar o sistema imunológico e a função cognitiva, causar problemas sociais, prejudicar a saúde mental e a capacidade de realizar seus afazeres habituais, bem como causar outros prejuízos 5 . Há indícios de que cerca de $20 \%$ desses pacientes se recusariam a continuar o tratamento se as náuseas e os vômitos não fossem controlados adequadamente 5 . Sendo assim, é importante que NVIQ sejam precocemente identificados e avaliados de modo a se intervir de forma eficaz por meio de manejo clínico correspondente.

Para tanto, faz-se necessário o uso de instrumentos que possibilitem a identificação e a monitorização de NVIQ e, assim, o planejamento de intervenções adequadas para essa condição. $\mathrm{O}$ uso de instrumentos de avaliação devidamente validados permite identificar de maneira objetiva alteraçôes físicas, fenômenos subjetivos e garantir o acompanhamento da progressão de um estado de saúde-doença, além de uniformizar a conduta a ser aplicada em cada situação ${ }^{6}$. A adaptação transcultural de um instrumento contribui para a diminuiçấo do tempo despendido com uma avaliação ampla e sem parâmetros objetivos e permite que resultados sejam comparados com outros contextos, de forma a se planejar estratégias efetivas de controle dos sintomas ${ }^{7}$.

Neste contexto, a escala Morrow Assessment of Nausea and Emesis (MANE), desenvolvida originalmente nos Estados Unidos da América, trata-se de um instrumento de rastreio que avalia a frequência, a gravidade e a duraçáo de náuseas e vômitos relacionados aos momentos pré e pós-quimioterapia ${ }^{8}$. Permite avaliar, também, o uso e a eficácia da medicação antiemética utilizada pelo paciente. Segundo Morrow ${ }^{9}$, náuseas e vômitos referem-se a sintomas subjetivos, o que permite o uso de medidas indiretas para sua avaliação.

De acordo com a literatura disponível até a realização deste estudo, não há no contexto brasileiro instrumentos que permitam a avaliação de NVIQ especificamente. Assim, este artigo tem por objetivo realizar a traduçáo e a adaptação transcultural da escala MANE para a cultura brasileira.

\section{MÉTODO}

Estudo com delineamento correlacional do tipo survey. O processo de tradução e adaptação cultural foi realizado de acordo com o protocolo da European Organization for Research and Treatment of Cancer (EORTC) - Quality of Life Group (QLG) ${ }^{10}$, seguindo as etapas descritas a seguir: - $1^{\text {a }}$ etapa: traduçáo do instrumento, do idioma de origem para o idioma-alvo, por dois tradutores independentes falantes nativos do idioma de origem (inglês) com um bom domínio do idioma-alvo (português), sem necessidade de que sejam tradutores profissionais.

- 2a etapa: as traduçóes independentes realizadas de acordo com o item anterior foram conciliadas e sintetizadas em uma única versão pelos pesquisadores, com o intuito de alcançar uma traduçáo ideal de cada item.

- $3^{a}$ etapa: a versão do instrumento conciliada e sintetizada foi traduzida novamente para o inglês por dois tradutores independentes com domínio do idioma de origem. Após essa etapa, as versôes traduzidas e retrotraduzidas foram encaminhadas para o primeiro autor da escala original.

- 4a etapa: a versão final da MANE foi submetida à avaliação de oito juízes (médicos e enfermeiros especialistas em oncologia), conforme preconizado pelo protocolo EORTC, para análise do conteúdo da escala, com vistas a avaliá-la quanto à clareza dos itens. Os juízes avaliaram a escala de forma geral e cada um dos itens individualmente.

- $5^{a}$ etapa: trata-se do teste-piloto, no qual o instrumento foi aplicado a um grupo de 15 pacientes para a verificação de aplicabilidade da versão adaptada da escala. Vale ressaltar que aqueles que participaram do teste-piloto não fizeram parte da amostra final deste estudo.

O presente estudo foi desenvolvido em uma clínica de oncologia do Sistema Único de Saúde (SUS) localizada no Sul do Estado de Minas Gerais. A clínica realiza atendimentos ambulatoriais e na modalidade de interconsulta, atendendo a 26 municípios integrantes de sua Regional. Ainda, oferece tratamento radioterápico, bem como conta com um setor de cuidados paliativos. 
De acordo com os dados do sistema de informação de Registro Hospitalar de Câncer, do Instituto Nacional de Câncer José Alencar Gomes da Silva (INCA) ${ }^{11}$, nessa clínica de oncologia, os cânceres de pele são os mais frequentes $(25,58 \%)$, seguido por cânceres nos órgãos genitais masculinos $(17,03 \%)$ e nos órgãos do aparelho digestivo (16,82\%).

Nesse serviço, o protocolo de medicação antiemética consiste no uso de medicaçóes como Ondansetrona, Bromoprida e Cimetidina, antes e após a realização da quimioterapia.

Este estudo foi aprovado pelo Comitê de Ética em Pesquisa da Universidade Federal de Alfenas (CAAE: 91681118.0.0000.5142; parecer: 2.815.937).

A população foi composta por pacientes com câncer em tratamento quimioterápico antineoplásico na clínica de oncologia supracitada, presentes no serviço durante o período de coleta de dados desta pesquisa.

A amostra foi por conveniência, do tipo não probabilística. Vale ressaltar que os participantes, como todos os demais pacientes atendidos na referida clínica, realizavam o protocolo padrão para prevenção de NVIQ.

Os critérios de inclusão foram: ter 18 anos ou mais de idade, ter diagnóstico de câncer (independentemente do tipo), estar em tratamento quimioterápico antineoplásico, ter condiçóes clínicas aceitáveis para a participação no estudo por meio das respostas aos instrumentos utilizados. Foram excluídos os pacientes que apresentassem um escore maior do que 3 na Eastern Cooperative Oncology Group (ECOG).

Os instrumentos a seguir foram utilizados no processo de adaptação transcultural da escala MANE para o Brasil.

\section{QUESTIONÁRIO PARA CARACTERIZAÇÃO SOCIODEMOGRÁFICA E CLÍNICA}

O questionário para avaliação das características sociodemográficas e clínicas foi desenvolvido pelos autores do estudo, com a finalidade de se investigar as seguintes variáveis: idade, sexo, nível de escolaridade e estado civil, tipo de câncer, tempo de tratamento quimioterápico antineoplásico, antieméticos em uso, e avaliação quanto à eficácia dos antieméticos prescritos para uso domiciliar.

\section{EASTERN COOPERATIVE ONCOLOGY GROUP}

Constitui-se de uma escala de avaliação de desempenho que, por meio de critérios padronizados, é capaz de mensurar as consequências de uma doença sobre as atividades de vida diária de uma pessoa (performance status). É capaz de descrever o nível funcional de uma pessoa em termos de sua capacidade de autocuidado, atividades de vida diária e capacidade física. A escala foi desenvolvida pelo ECOG, agora parte do ECOG-ACRIN
Cancer Research Group, e publicada em 1982, sendo uma escala de domínio público ${ }^{12,13}$.

Seus escores variam de 0 a 5 , sendo que, quanto maior a pontuação, pior é o nível funcional do paciente. Assim, o escore 0 significa que a pessoa está totalmente ativa, capaz de realizar todas suas atividades sem restrição; o escore 1 destina-se a pessoas com restrição de atividades extenuantes, mas é capaz de deambular e realizar atividades leves, como trabalho doméstico e de escritório; o escore 2 caracteriza pessoas incapazes de realizar atividades laborais, mas deambula e possui autocuidado presente; o escore 3 apresenta autocuidado limitado e, em mais de $50 \%$ dos momentos de vigília, está confinado à cadeira ou ao leito; o escore 4 é destinado a pessoas totalmente confinadas ao leito, com impossibilidade de autocuidado; e o escore 5 para caracterizar a morte ${ }^{12}$.

\section{ESCALA VISUAL NUMÉRICA}

A escala visual numérica (EVN) foi utilizada para a avaliação da intensidade das náuseas e vômitos. Trata-se de um instrumento amplamente utilizado para mensurar a intensidade de náuseas (EVN-N) e vômitos $(\mathrm{EVN}-\mathrm{V})$ induzidos por quimioterapia, com marcaçôes numéricas uniformemente espaçadas ( 0 a 10$)$, de modo que, quanto maior o número, maior a intensidade do sintoma avaliado ${ }^{14}$. Essas escalas geralmente são de fácil entendimento e interpretação tanto para pacientes quanto para clínicos e pesquisadores ${ }^{15}$. De acordo com a literatura, a EVN é capaz de diferenciar, de maneira confiável, mudanças quanto à gravidade de náusea e vômito ${ }^{16}$.

\section{ESCALA MORROW ASSESSMENT OF NAUSEA AND EMESIS}

A escala MANE é uma ferramenta que possibilita a avaliação de aspectos relacionados à NVIQ. É um instrumento composto por 16 itens breves, os quais estão distribuídos nas seguintes temáticas acerca de NVIQ: frequência, gravidade e duração desses eventos nos momentos pré e pós-quimioterapia antineoplásica ${ }^{8}$. Considerando a forma como os resultados são trabalhados pelo autor da escala original, sem um modelo dimensional que fundamenta o instrumento, a escala não apresenta um escore final, de forma que se deve proceder à análise individual de cada um dos itens da escala, interpretando-os de acordo com a individualidade de cada paciente.

A coleta de dados foi realizada na sala de espera da clínica de oncologia, no setor de quimioterapia, onde os possíveis participantes eram abordados e convidados a participar do estudo. Após consentimento formal, por meio da assinatura do Termo de Consentimento Livre e Esclarecido (TCLE), foi iniciada a coleta de dados com a aplicaçáo dos instrumentos. De acordo com Morrow ${ }^{8}$, o instrumento pode ser autoaplicável, no entanto, optou- 
-se por realizar a coleta de dados por meio de entrevista realizada pelas próprias pesquisadoras (GMI, ACGF, EMCP). Essa escolha se deu pelo fato de que, como identificado no teste-piloto, a maior parte dos pacientes possuía nível de escolaridade baixo e/ou dificuldades para o preenchimento dos instrumentos, como por exemplo, em razão de problemas visuais. Para se evitar possíveis vieses relacionados à coleta de dados por meio de entrevista, as pesquisadoras foram capacitadas para a realização dessa atividade, de modo a serem realizadas de forma padronizada, apenas procedendo à leitura dos itens, sem interpretá-los.

Os instrumentos utilizados neste estudo foram digitalizados por meio da plataforma Google Forms (serviço gratuito para a criação de formulários on-line), assim, o registro dos dados foi feito de forma on-line. A aplicação dos questionários utilizados no estudo durou em média 20 minutos.

Para avaliação da concordância entre os juízes, no processo de traduçáo e adaptação transcultural da MANE para o português do Brasil, foi utilizado o índice de validação de conteúdo (IVC). Os itens que apresentaram IVC abaixo ou igual a $75 \%$ passaram por uma readequação, conforme as sugestôes propostas pelos juízes $^{17,18}$. Após as adequaçôes sugeridas, os itens foram readequados e enviados novamente para avaliação dos juízes. Foram calculados um IVC para a escala geral e um para cada item separadamente.

Os dados de caracterização da amostra foram analisados por meio de estatísticas descritivas (frequência e percentual válido). As evidências de validade foram baseadas na relação dos itens da MANE com as variáveis externas. Análises de correlação multimétodos foram realizadas para estimar o nível de associação entre os itens da escala MANE e os escores das EVN-V e EVN-N ao nível de $\mathrm{p}<0,05$.

A correlação de Spearman foi utilizada para o estabelecimento da correlação entre variáveis categóricas ordenadas; a de Pearson para a correlação entre as variáveis métricas das escalas numéricas. A força da associação dada pelo coeficiente estimado foi interpretada da seguinte maneira: - associação perfeita; 0,9 a 0,7 - associação forte; 0,6 a 0,4 - associação moderada; 0,3 a 0,1 - associação fraca; 0 - nenhuma associação ${ }^{19}$.

\section{RESULTADOS}

A amostra foi constituída por 160 pacientes com idade média de 65,37 anos ( $\mathrm{DP}=12,19)$. O tempo médio de quimioterapia antineoplásica foi de 23 meses $(\mathrm{DP}=32,52)$. Os demais dados referentes às características sociodemográficas e clínicas estão descritos na Tabela 1 .
Tabela 1. Características sociodemográficas e clínicas dos participantes

\begin{tabular}{|c|c|c|}
\hline Varióveis & $\mathbf{N}$ & $\%$ \\
\hline \multicolumn{3}{|l|}{ Sexo } \\
\hline Feminino & 58 & 36,3 \\
\hline Masculino & 102 & 63,7 \\
\hline \multicolumn{3}{|l|}{ Estado civil } \\
\hline Solteiro(a) & 24 & 15,0 \\
\hline Casado(a) & 102 & 63,7 \\
\hline Viúvo(a) & 17 & 10,6 \\
\hline Amasiado(a) & 6 & 3,8 \\
\hline $\begin{array}{l}\text { Divorciado(a) sem outro(a) } \\
\text { companheiro(a) }\end{array}$ & 7 & 4,4 \\
\hline $\begin{array}{l}\text { Divorciado(a) com outro(a) } \\
\text { companheiro(a) }\end{array}$ & 4 & 2,5 \\
\hline \multicolumn{3}{|l|}{ Nível de escolaridade } \\
\hline Analfabeto: sabe ler e escrever & 29 & 18,1 \\
\hline $\begin{array}{l}\text { Baixa (ensino fundamental } \\
\text { completo e incompleto) }\end{array}$ & 94 & 58,8 \\
\hline $\begin{array}{l}\text { Média (ensino médio completo e } \\
\text { incompleto) }\end{array}$ & 25 & 15,6 \\
\hline $\begin{array}{l}\text { Alta (ensino superior completo e } \\
\text { incompleto) }\end{array}$ & 12 & 7,5 \\
\hline \multicolumn{3}{|l|}{ Tipo de câncer } \\
\hline Próstata & 44 & 27,5 \\
\hline Mama & 22 & 13,8 \\
\hline Intestino & 20 & 12,5 \\
\hline Outros & 74 & 46,2 \\
\hline
\end{tabular}

As traduçóes do idioma de origem para o idioma-alvo apresentaram pequenas discrepâncias; a primeira apresentou palavras em seu sentido literal, enquanto a segunda utilizou de palavras e expressóes comuns ao português brasileiro. As duas versões foram sintetizadas em uma única escala, processo realizado pelos pesquisadores. As retrotraduçóes feitas a partir da versão unificada da escala não apresentaram diferenças significativas. Ao final do processo de tradução, as versóes retrotraduzidas da escala foram encaminhadas para o autor original da escala MANE, o qual garantiu equivalência conceitual. Os itens $1,2,4,5,7,9,10,12,13,15$ e 16 apresentaram IVC acima de $75 \%$, sendo assim, náo houve necessidade de reformulação. Os demais foram reformulados de acordo com as sugestōes dos juízes. Em relação ao título da escala, $87,5 \%$ dos juízes foram favoráveis à "escala para avaliação de náusea e vômito relacionados à quimioterapia".

Quanto ao teste-piloto, segundo sugestóes dos pacientes, nos itens 4, 7, 8, 10 e 13, foram incluídas as opçôes "Não senti náusea" ou "Não tive vômito", a 
depender do sintoma abordado no item. Ainda, mais de 90\% dos respondentes consideraram os itens da escala como claros, de fácil entendimento e não ofensivos. A Tabela 2 apresenta os itens da escala de acordo com a característica a ser avaliada.

Quanto à EVN, a média do escore para intensidade da náusea foi de 3,03 (DP=3,49); para a avaliação da intensidade dos episódios de vômito, a média do escore foi de $1,72(\mathrm{DP}=3,18)$, resultados que corroboram o identificado pela aplicação da escala MANE, uma vez que a maior parte dos respondentes não sentiu náusea e/ou vômito ou, quando presentes, esses sintomas eram de baixa frequência, duração ou gravidade (Tabela 3 ).

Tabela 2. Distribuição dos itens da escala Morrow Assessment of Nausea and Emesis de acordo com as características de avaliação

\begin{tabular}{lcccc} 
& $\begin{array}{c}\text { Rastreio } \\
\text { (sim ou não) }\end{array}$ & $\begin{array}{c}\text { Frequência } \\
\text { (escala ordinal } \\
\text { de 5 pontos) }\end{array}$ & $\begin{array}{c}\text { Intensidade } \\
\text { (escala ordinal } \\
\text { de 8 pontos) }\end{array}$ & $\begin{array}{c}\text { Duração } \\
\text { (escala ordinal } \\
\text { de 8 pontos) }\end{array}$ \\
\hline $\begin{array}{l}\text { Antes da QT } \\
\text { Náusea } \\
\text { Vômito }\end{array}$ & Item 9 & Item 10 & Item 11 \\
$\begin{array}{l}\text { Depois da QT } \\
\text { Item } 12\end{array}$ & Item 13 & Item 14 \\
$\begin{array}{l}\text { Náusea } \\
\text { Vômito }\end{array}$ & Item 1 & Item 2 & Item 3 & Item 4 \\
Medicação antiemética & Item 5 & Item 6 & Item 7 & Item 8 \\
\hline
\end{tabular}

Legenda: QT = Quimioterapia

Tabela 3. Apresentação dos resultados da escala Morrow Assessment of Nausea and Emesis

\begin{tabular}{|c|c|c|c|}
\hline Itens da escala MANE & Categorias de resposta & Freq. & $\%$ \\
\hline \multirow{2}{*}{$\begin{array}{l}\text { 1. Você sentiu náusea depois da sua } \\
\text { última quimioterapia? Marque uma } \\
\text { das opções }\end{array}$} & Sim & 47 & 29,4 \\
\hline & Não & 113 & 70,6 \\
\hline \multirow{5}{*}{$\begin{array}{l}\text { 2. Quantas vezes você sentiu náusea } \\
\text { depois da quimioterapia? }\end{array}$} & Nenhuma vez & 90 & 56,3 \\
\hline & 1 a 3 vezes & 37 & 23,1 \\
\hline & 4 a 6 vezes & 9 & 5,6 \\
\hline & 7 a 9 vezes & 2 & 1,3 \\
\hline & 9 ou mais vezes & 22 & 13,8 \\
\hline \multirow{6}{*}{$\begin{array}{l}\text { 3. Como foi a sua pior sensação } \\
\text { de náusea depois da sua última } \\
\text { quimioterapia? Marque uma das } \\
\text { opções }\end{array}$} & Muito fraca & 91 & 56,9 \\
\hline & Fraca & 15 & 9,4 \\
\hline & Moderada & 20 & 12,5 \\
\hline & Forte & 13 & 8,1 \\
\hline & Muito forte & 13 & 8,1 \\
\hline & Insuportável & 8 & 5,0 \\
\hline \multirow{8}{*}{$\begin{array}{l}\text { 4. Quando foi sua pior sensação de } \\
\text { náusea? Marque uma das opções }\end{array}$} & Não senti náusea & 88 & 55,0 \\
\hline & Durante a quimioterapia & 8 & 5,0 \\
\hline & 0 a 4 horas depois da quimioterapia & 26 & 16,3 \\
\hline & 5 a 8 horas depois da quimioterapia & 6 & 3,8 \\
\hline & 9 a 12 horas depois da quimioterapia & 5 & 3,1 \\
\hline & 13 a 24 horas depois da quimioterapia & 5 & 3,1 \\
\hline & Mais de 24 horas depois da quimioterapia & 18 & 11,2 \\
\hline & A sensação de náusea se manteve igual o tempo todo & 4 & 2,5 \\
\hline
\end{tabular}


Tabela 3. continuação

Itens da escala MANE
5. Você teve vômito depois da sua
última quimioterapia? Marque uma
das opções

7. Como foi o pior vômito que você já teve depois da sua última quimioterapia? Marque uma das opções

\begin{tabular}{|c|c|c|}
\hline Categorias de resposta & Freq. & $\%$ \\
\hline Sim & 15 & 9,4 \\
\hline Não & 145 & 90,6 \\
\hline Nenhuma vez & 126 & 78,8 \\
\hline 1 a 3 vezes & 16 & 10,0 \\
\hline 4 a 6 vezes & 5 & 3,1 \\
\hline 7 a 9 vezes & 2 & 1,2 \\
\hline 9 ou mais vezes & 11 & 6,9 \\
\hline Não tive vômito & 125 & 78,1 \\
\hline Muito fraco & 2 & 1,2 \\
\hline Fraco & 3 & 1,9 \\
\hline Moderado & 11 & 6,9 \\
\hline Forte & 9 & 5,6 \\
\hline Muito forte & 6 & 3,8 \\
\hline Insuportável & 4 & 2,5 \\
\hline Não tive vômito & 126 & 78,8 \\
\hline Durante a quimioterapia & 2 & 1,2 \\
\hline 0 a 4 horas depois da quimioterapia & 14 & 8,8 \\
\hline 5 a 8 horas depois da quimioterapia & 3 & 1,9 \\
\hline 9 a 12 horas depois da quimioterapia & 4 & 2,5 \\
\hline 13 a 24 horas depois da quimioterapia & 1 & 0,6 \\
\hline Mais de 24 horas depois da quimioterapia & 8 & 5,0 \\
\hline O vômito se manteve igual o tempo todo & 2 & 1,2 \\
\hline Sim & 14 & 8,8 \\
\hline Não & 146 & 91,2 \\
\hline Não senti náusea & 145 & 90,6 \\
\hline Muito fraca & 1 & 0,6 \\
\hline Fraca & 3 & 1,9 \\
\hline Moderada & 7 & 4,4 \\
\hline Forte & 2 & 1,3 \\
\hline Muito forte & 1 & 0,6 \\
\hline Insuportável & 1 & 0,6 \\
\hline Não senti náusea antes da última quimioterapia & 146 & 91,3 \\
\hline $\begin{array}{l}\text { Senti náusea } 1 \text { a } 3 \text { horas antes da última } \\
\text { quimioterapia }\end{array}$ & 7 & 4,4 \\
\hline $\begin{array}{l}\text { Senti náusea } 4 \text { a } 6 \text { horas antes da última } \\
\text { quimioterapia }\end{array}$ & 1 & 0,6 \\
\hline $\begin{array}{l}\text { Senti náusea } 7 \text { a } 9 \text { horas antes da última } \\
\text { quimioterapia }\end{array}$ & 1 & 0,6 \\
\hline $\begin{array}{l}\text { Senti náusea } 9 \text { horas ou mais antes da última } \\
\text { quimioterapia }\end{array}$ & 5 & 3,1 \\
\hline
\end{tabular}

11. Quanto tempo antes de sua última quimioterapia você sentiu náusea?
8. Quando foi o pior vômito que você já teve? Marque uma das opções

10. Como foi sua sensação de náusea antes da quimioterapia? Marque uma das opções

9. Você sentiu náusea antes da sua última quimioterapia? Marque uma das opções

11. Quanto tempo antes de sua última
quimioterapia você sentiu náusea?


Tabela 3. continuação

\begin{tabular}{|c|c|c|c|}
\hline Itens da escala MANE & Categorias de resposta & Freq. & $\%$ \\
\hline \multirow{2}{*}{$\begin{array}{l}\text { 12. Você teve vômitos antes da sua } \\
\text { última quimioterapia? Marque uma } \\
\text { das opções }\end{array}$} & Sim & 3 & 1,9 \\
\hline & Não & 157 & 98,1 \\
\hline \multirow{6}{*}{$\begin{array}{l}\text { 13. Como foi o pior vômito que } \\
\text { você já teve antes da sua última } \\
\text { quimioterapia? Marque uma das } \\
\text { opções }\end{array}$} & Não tive vômito & 156 & 97,6 \\
\hline & Muito fraco & 1 & 0,6 \\
\hline & Fraco & 1 & 0,6 \\
\hline & Forte & 1 & 0,6 \\
\hline & Muito forte & 1 & 0,6 \\
\hline & Insuportável & 0 & 0 \\
\hline \multirow{5}{*}{$\begin{array}{l}\text { 14. Quanto tempo antes da sua última } \\
\text { quimioterapia você teve vômito? }\end{array}$} & Não vomitei antes da última quimioterapia & 157 & 98,1 \\
\hline & Vomitei 1 a 3 horas antes da última quimioterapia & 2 & 1,3 \\
\hline & Vomitei 4 a 6 horas antes da última quimioterapia & 1 & 0,6 \\
\hline & Vomitei 7 a 9 horas antes da última quimioterapia & 0 & 0 \\
\hline & $\begin{array}{l}\text { Vomitei } 9 \text { horas ou mais antes da última } \\
\text { quimioterapia }\end{array}$ & 0 & 0 \\
\hline \multirow{2}{*}{$\begin{array}{l}\text { 15. Você tomou alguma medicação } \\
\text { para náuseas e/ou vômitos em sua } \\
\text { última quimioterapia? Marque uma } \\
\text { das opções }\end{array}$} & Sim & 36 & 22,5 \\
\hline & Não & 124 & 77,5 \\
\hline \multirow{4}{*}{ 16. Esta medicação foi útil? } & Sim & 34 & 21,2 \\
\hline & Pouco & 4 & 2,5 \\
\hline & Muito pouco & 3 & 1,9 \\
\hline & Não & 119 & 74,4 \\
\hline
\end{tabular}

Legendas: MANE = Escala Morrow Assessment of Nausea and Emesis; Freq. = frequência.

De acordo com a Tabela 4, ao correlacionar os itens da escala MANE com a EVN-N e EVN-V (correlação ponto bisserial e de Spearman), foram obtidos coeficientes de correlação altamente significativos $(\mathrm{p}<0,01)$ e significativos $(\mathrm{p}<0,05)$, o que indica convergência entre os dois métodos de avaliação de náusea e vômito (EVN e MANE). Os itens entre 1 e 8 da escala MANE apresentaram associaçóes de forte a moderada com as escalas EVN-N e EVN-V (Tabela 4). Tais itens correspondem à avaliação dos sintomas de náusea e vômito depois da quimioterapia. Quanto aos itens de 9 a 16 da escala MANE, a correlaçáo foi fraca em relação à EVN-N e EVN-V (Tabela 3). Os itens de 9 a 14 correspondem à avaliaçáo dos sintomas de náusea $\mathrm{e}$ vômito antes da quimioterapia; os itens 15 e 16 referem-se ao uso de medicaçáa antiemética e à utilidade desta quanto a esses sintomas.

A interpretaçáo dos resultados provenientes da correlação se deu da seguinte forma: por exemplo, a relação entre a EVN-N e o item 1 da escala MANE foi de $-0,54$ (negativa) (Tabela 4), indicando que os participantes que marcaram os maiores números na $\mathrm{EVN}-\mathrm{N}$ foram os que marcaram o menor número no item 1 da escala MANE (neste caso, $1=\operatorname{sim}$, indicando que sentiu náusea depois da quimioterapia) e assim por diante. Para o caso das relaçóes positivas, tomando como exemplo a relação entre o item 2 da escala MANE e a EVN-N ( $r=0,77)$, a interpretação é que quanto maior o número marcado na $\mathrm{EVN}-\mathrm{N}$, maior o número marcado no item 2 da escala MANE (ou seja, maior a frequência de náusea antes da quimioterapia).

\section{DISCUSSÃO}

Haja vista a escassez de instrumentos com a finalidade de avaliar NVIQ, faz necessário traduzir e adaptar a escala MANE para a cultura brasileira. De acordo com Morrow ${ }^{8}$, trata-se de uma ferramenta que permite ao profissional de saúde uma avaliação ampla e objetiva dessas manifestaçôes, podendo auxiliar a tomada de decisão clínica para o seu respectivo controle.

Por meio de um método sistemático de tradução e adaptação cultural, é possível adequar um instrumento elaborado em uma outra cultura e outro idioma a uma 
Tabela 4. Matriz de correlação multimétodos entre os itens da escala e as escalas numéricas de intensidade de náuseas e vômitos

\begin{tabular}{|c|c|c|c|c|c|c|c|c|c|c|c|c|c|c|c|c|c|}
\hline & EVN-N & EVN-V & 1 & 2 & 3 & 4 & 5 & 6 & 7 & 8 & 9 & 10 & 11 & 12 & 13 & 14 & 15 \\
\hline MANE & $0,68^{* * \neq}$ & & & & & & & & & & & & & & & & \\
\hline 1 & $-0,54^{* *}$ & $-0,33^{* *}$ & & & & & & & & & & & & & & & \\
\hline 2 & $0,77^{* *}$ & $0,56^{* *}$ & $-0,69^{* *}$ & & & & & & & & & & & & & & \\
\hline 3 & $0,79^{* *}$ & $0,53^{* *}$ & $-0,61^{* *}$ & $0,89^{* *}$ & & & & & & & & & & & & & \\
\hline 4 & $0,73^{* *}$ & $0,40^{* *}$ & $-0,65^{* *}$ & $0,89^{* *}$ & $0,87^{* *}$ & & & & & & & & & & & & \\
\hline 5 & $-0,33^{* *}$ & $-0,57^{* *}$ & $0,33^{* *}$ & $-0,24^{* *}$ & $-0,31^{* *}$ & $-0,19 *$ & & & & & & & & & & & \\
\hline 6 & $0,60^{* *}$ & $0,83^{* *}$ & $-0,27^{* *}$ & $0,57^{* *}$ & $0,59 * *$ & $0,43^{* *}$ & $-0,58^{* *}$ & & & & & & & & & & \\
\hline 7 & $0,61^{* *}$ & $0,80^{* *}$ & $-0,22^{* *}$ & $0,54^{* *}$ & $0,60^{* *}$ & $0,41^{* *}$ & $-0,56^{* *}$ & $0,95^{* *}$ & & & & & & & & & \\
\hline 8 & $0,57^{* *}$ & $0,79^{* *}$ & $-0,22^{* *}$ & $0,54^{* *}$ & $0,57^{* *}$ & $0,42^{* *}$ & $-0,56^{* *}$ & $0,95^{* *}$ & $0,97^{* *}$ & & & & & & & & \\
\hline 9 & $-0,26^{* *}$ & $-0,16^{*}$ & $0,43^{* *}$ & $-0,31^{* *}$ & $-0,31^{* *}$ & $-0,29 * *$ & $0,28^{* *}$ & $-0,25^{* *}$ & $-0,22^{* *}$ & $-0,23^{* *}$ & & & & & & & \\
\hline 10 & $0,32^{* *}$ & $0,19^{*}$ & $-0,41^{* *}$ & $0,36^{* *}$ & $0,37^{* *}$ & $0,33^{* *}$ & $-0,27^{* *}$ & $0,32^{* *}$ & $0,29 * *$ & $0,30^{* *}$ & $-0,90^{* *}$ & & & & & & \\
\hline 11 & $0,30^{* *}$ & $0,16^{*}$ & $-0,43^{* *}$ & $0,36^{* *}$ & $0,36 * *$ & $0,33^{* *}$ & $-0,27^{* *}$ & $0,30^{* *}$ & $0,26^{* *}$ & $0,27^{* *}$ & $-0,92^{* *}$ & $0,97^{* *}$ & & & & & \\
\hline 12 & $-0,20^{*}$ & $-0,19^{*}$ & 0,11 & $-0,11$ & $-0,10$ & $-0,06$ & 0,11 & $-0,28^{* *}$ & $-0,28^{* *}$ & $-0,31^{* *}$ & $0,28^{* *}$ & $-0,28^{* *}$ & $-0,30^{* *}$ & & & & \\
\hline 13 & $0,23^{* *}$ & $0,24^{* *}$ & $-0,07$ & $0,17^{*}$ & 0,15 & 0,09 & $-0,09$ & $0,34^{* *}$ & $0,32^{* *}$ & $0,33^{* *}$ & $-0,23^{* *}$ & $0,23^{* *}$ & $0,25^{* *}$ & $-0,86^{* *}$ & & & \\
\hline 14 & $0,19^{*}$ & $0,21^{* *}$ & $-0,11$ & 0,11 & 0,10 & 0,06 & $-0,12$ & $0,28^{* *}$ & $0,28^{* *}$ & $0,31^{* *}$ & $-0,28^{* *}$ & $0,28^{* *}$ & $0,30^{* *}$ & $-1,00$ & $0,86^{* *}$ & & \\
\hline 15 & $-0,37^{* *}$ & $-0,25^{*}$ & $0,44^{* *}$ & $-0,38^{* *}$ & $-0,43^{* *}$ & $-0,43^{* *}$ & 0,14 & $-0,16^{*}$ & $-0,21^{* *}$ & $-0,15$ & $0,26^{* *}$ & $-0,29 * *$ & $-0,26 * *$ & 0,04 & $-0,01$ & $-0,04$ & \\
\hline 16 & $-0,31^{* *}$ & $-0,23^{* *}$ & $0,36^{* *}$ & $-0,32^{* *}$ & $-0,36^{* *}$ & $-0,37^{* *}$ & 0,08 & $-0,13^{*}$ & $-0,17^{*}$ & $-0,11$ & $0,22^{* *}$ & $-0,25^{* *}$ & $-0,22^{* *}$ & 0,03 & $-0,07$ & $-0,03$ & $0,91^{* *}$ \\
\hline
\end{tabular}

Legendas: $\mathrm{MANE}=$ Escala Morrow Assessment of Nausea and Emesis; EVN-N = Escala visual numérica para mensurar a intensidade de náuseas; EVN-V = Escala visual numérica para mensurar a intensidade do vômito.

$\left({ }^{*}\right) \mathrm{p}<0,05$.

$(* *) \mathrm{p}<0,01$.

${ }^{*}$ ) Correlação de Pearson (correlaçấo entre as variáveis métricas das escalas numéricas)

Nota: Correlaçấo ponto bisserial (correlação entre variáveis dicotômicas [itens 1, 5, 9, 12 e 15] de rastreio e variável métrica das escalas numéricas); correlação de Spearman (correlação entre variáveis categóricas ordenadas [itens que avaliam frequência de náuseas e vômitos depois e antes da quimioterapia = 2 e 6 ; intensidade $=3,10,7$ e 13 ; e duração $=4,11,8$ e 14]).

nova realidade, de acordo com suas peculiaridades. A adaptação transcultural consiste em um processo que analisa a linguagem por meio da traduçâo e estuda questóes relacionadas à cultura-alvo, sendo um pré-requisito para utilizaçáo em estudos realizados com o mesmo instrumento em diferentes culturas ${ }^{20}$.

NVIQ podem causar impactos negativos na saúde física e emocional da pessoa com câncer, diminuindo sua qualidade de vida. Portanto, avaliar corretamente esses efeitos colaterais é uma necessidade para a melhoria dos cuidados clínicos ${ }^{21,22}$. Em relação à NVIQ, fatores como menor idade, histórico de náuseas relacionadas à gestação, menos horas de sono na noite que precede à quimioterapia, estágio mais avançado do tumor e potencial emetogênico do quimioterápico em uso podem aumentar a frequência e a gravidade desses sintomas ${ }^{23}$.

A baixa frequência de NVIQ nos pacientes deste estudo pode ser justificada pelo uso de protocolo com medicações antieméticas desde o início do tratamento quimioterápico, no serviço onde os dados foram coletados. Vale ressaltar que indivíduos que possuem prescrição de protocolo antiemético, baseado em diretrizes disponíveis na literatura, apresentam menor incidência de NVIQ do que aqueles que possuem uma prescrição inconsistente ${ }^{24}$. Esta é uma medida importante como estratégia de prevenção e controle desses sintomas, considerando a qualidade de vida e adesão da pessoa ao tratamento $^{24,25}$.

A avaliação de NVIQ por meio de instrumentos bem estruturados tornou-se uma prioridade tanto em pesquisas científicas quanto na prática clínica, uma vez que pode auxiliar a compreensão do efeito das terapias antieméticas no alívio dos sintomas, a subsidiar informações para uso epidemiológico, a melhorar o relato e a comunicação entre equipes, além de contribuir para uma melhor assistência às pessoas com câncer ${ }^{6,26}$. Entretanto, o caráter subjetivo, principalmente das náuseas, e a dificuldade de comunicação desses sintomas entre pacientes e profissionais de saúde ainda constituem um desafio para sua avaliação e controle ${ }^{26}$.

De acordo com os resultados do presente estudo, as escalas MANE, EVN-N e EVN-V apresentaram-se como 
métodos de avaliação convergentes entre si. Os itens da MANE responsáveis por avaliar NVIQ no momento pós-quimioterapia apresentaram associaçôes forte e moderada com as escalas EVN-N e EVN-V, sinalizando a capacidade da escala MANE em identificar a intensidade desses sintomas. A escala MANE e as EVN são consideradas de fácil administração e possuem um alto grau de aceitação dos pacientes ${ }^{27}$. No entanto, de acordo com dados da literatura nacional, baixos níveis de escolaridade podem dificultar a interpretação de informaçôes ${ }^{28}$.

Ambas as avaliaçôes são úteis e exigem pouco tempo de aplicação, além de permitirem valores numéricos elegíveis para codificação em análises estatísticas ${ }^{8}$. No entanto, as EVN permitem a avaliaçáo apenas da intensidade dessas condiçóes, deixando de contemplar aspectos referentes ao seu período de ocorrência, ou ainda, deixam de contemplar náuseas e vômitos em fase antecipatória ${ }^{26}$. Náuseas e vômitos antecipatórios são sintomas e sinais amplamente assimilados à experiência quimioterápica prévia, sendo que há risco da sua exacerbação à medida que o número de ciclos de quimioterapia aumenta. Sendo assim, sons, cheiros e imagens desconhecidos podem ser ligados à experiência de náusea e vômito subsequente, desencadeando novamente essas manifestaçóes antes mesmo da administração do quimioterápico ${ }^{29}$.

Quanto à avaliação de náusea e vômito antes da quimioterapia, as correlaçóes entre as escalas MANE e EVN foram fracas. De acordo com Morrow ${ }^{8}$, náuseas e vômitos antecipatórios são fenômenos que apresentam maior variação quanto à frequência, à duração e à severidade, sendo menos frequentes entre os pacientes ${ }^{9}$, assim como observado no presente estudo, o que pode explicar a baixa correlação. Em um estudo realizado com mulheres com câncer de mama em uso de quimioterápicos moderadamente emetogênicos, verificou-se que apenas $11,90 \%$ das participantes apresentaram êmese antecipatória ${ }^{30}$.

Também houve correlação fraca entre as escalas EVN-N e EVN-V e os itens sobre o uso de medicação antiemética e sua utilidade da escala MANE. Possivelmente, isto pode ser explicado pelo fato de que as escalas visuais numéricas utilizadas neste estudo foram empregadas com o objetivo de avaliar exclusivamente a intensidade dos sintomas, sem o intuito de avaliar outras condiçôes, como por exemplo, a eficácia das medicaçôes utilizadas.

A realização deste estudo, em um único centro de oncologia, pode limitar a generalizaçáo dos resultados, considerando que o Brasil é um país com ampla diversidade cultural e sociodemográfica. Assim, estudos futuros podem empenhar-se em avaliar e adaptar culturalmente a escala em outros contextos regionais e populacionais, com o intuito de viabilizar sua validade externa. Ainda, a realização da coleta de dados por meio de entrevista pode ser caracterizada com uma possível limitação. No entanto, vale ressaltar que limitaçôes físicas decorrentes da idade, da doença em si e do próprio tratamento dificultaram o processo de resposta por parte dos participantes, os quais faziam a opção pela realização da entrevista.

Finalmente, a escala MANE pode auxiliar na melhoria da assistência em saúde a pessoas com câncer em quimioterapia antineoplásica, visto que possibilita a avaliação objetiva e o monitoramento de náuseas e vômitos por meio de uma abordagem multidimensional. Ainda, pode ser utilizada na realização de pesquisas científicas, como por exemplo, em estudos experimentais sobre intervençóes para controle de NVIQ.

\section{CONCLUSÃO}

A escala MANE apresenta-se como um instrumento adequado para a avaliação de NVIQ no contexto brasileiro. Essa é uma escala que permite uma ampla avaliação de NVIQ, possibilitando avaliá-los como fenômenos distintos em momentos também distintos, desde antes até após a realização da quimioterapia antineoplásica. A utilização de tal instrumento poderá contribuir para maior qualidade de assistência em saúde a pessoas acometidas por esses sinais e sintomas, bem como para a realização de pesquisas científicas sobre a temática.

\section{CONTRIBUIÇÕES}

Geovanna Maria Isidoro, Ana Cristina Gonçalves Ferreira, Eliza Mara das Chagas Paiva e Ana Cláudia Mesquita Garcia contribuíram substancialmente na concepçáo e/ou planejamento do estudo; na obtençáo, análise e/ou interpretaçáo dos dados. Jodi De Hunt Ferreira do Amaral e Everson Cristiano de Abreu Meireles contribuíram na redação e revisão crítica. Todos os autores aprovaram a versão final a ser publicada.

\section{DECLARAÇÃO DE CONFLITO DE INTERESSES}

Nada a declarar.

\section{FONTES DE FINANCIAMENTO}

Não há.

\section{REFERÊNCIAS}

1. World Health Organization. WHO report on cancer: setting priorities, investing wisely and providing care for all. Geneva: WHO; 2020.

2. Instituto Nacional de Câncer José Alencar Gomes da Silva. Estimativa 2020: incidência de câncer no Brasil 
[Internet]. Rio de Janeiro: INCA; 2019 [acesso 2020 nov 23]. Disponível em: https://www.inca.gov.br/sites/ ufu.sti.inca.local/files/media/document/estimativa-2020incidencia-de-cancer-no-brasil.pdf

3. Reis M. Farmacogenética aplicada ao câncer. Quimioterapia individualizada e especificidade molecular. Medicina (Ribeiráo Preto). 2006;39(4):57786. doi: https://doi.org/10.11606/issn.2176-7262. v39i4p577-586

4. Wang W, Lou G, Zhang Y. Olanzapine with ondansetron and dexamethasone for the prevention of cisplatin-based chemotherapy-induced nausea and vomiting in lung câncer. Medicine (Baltimore). 2018;97(37):e12331. doi: https://doi.org/10.1097/ MD.0000000000012331

5. Karimi S, Makhsosi BR, Seyedi-Andi SJ, et al. Surveying the effect of a self-care education program on severity of nausea and emesis in colorectal cancer patients under chemotherapy. J Multidiscip Healthc. 2017;10:301-7. doi: https://doi.org/10.2147/JMDH.S131111

6. Gardona RGB, Barbosa DA. The importance of clinical practice supported by health assessment tools. Rev Bras Enferm. 2018;71(4):1815-6. doi: https://doi. org/10.1590/0034-7167-2018710401

7. Epstein J, Osborne RH, Elsworth GR, et al. Crosscultural adaptation of the Health Education Impact Questionnaire: experimental study showed expert committee, not back-translation, added value. J Clin Epidemiol. 2015;68(4):360-9. doi: https://doi. org/10.1016/j.jclinepi.2013.07.013

8. Morrow GR. A patient report measure for the quantification of chemotherapy induced nausea and emesis: psychometric properties of the Morrow assessment of nausea and emesis (MANE). Br J Cancer Suppl. 1992;19:S72-4.

9. Morrow GR. Methodology in behavioral and psychosocial cancer research. The assessment of nausea and vomiting. Past problems, current issues and suggestions for future research. Cancer. 1984;53(10 Suppl):2267-80.

10. Kulis D, Bottomley A, Velikova G, et al. EORTC Quality of life group translation procedure. 4th ed. Brussels: EORTC; 2017.

11. Instituto Nacional de Câncer José Alencar Gomes da Silva. Integrador RHC: registro hospitalar de câncer [Internet]. Versão 3.2. Rio de Janeiro: INCA. $2012 \mathrm{dez}$ 14 - [acesso 2020 abr 3]. Disponível em: https://bit. ly/38MyrBF

12. ECOG-ACRIN Cancer Research Group [Internet]. Philadelphia, PA: ECOG_AGRIN; c2012-2020. ECOG performance status; [cited 2020 Aug 17]. Available from: https://ecog-acrin.org/resources/ecog-performance-status

13. Oken MM, Creech RH, Tormey DC, et al. Toxicity and response criteria of the Eastern Cooperative Oncology Group. Am J Clin Oncol. 1982;5(6):649-55.
14. Wood JM, Chapman K, Eilers J. Tools for assessing nausea, vomiting, and retching: a literature review. Cancer Nurs. 2011;34(1):E14-24. doi: https://doi. org/10.1097/ncc.0b013e3181e2cd79

15. Jaeschke R, Singer J, Guyatt GH. A comparison of sevenpoint and visual analogue scales. Data from a randomized trial. Control Clin Trials. 1990;11(1):43-51. doi: https:// doi.org/10.1016/0197-2456(90)90031-v

16. Meek R, Egerton-Warburton D, Mee MJ, et al. Measurement and monitoring of nausea severity in emergency department patients: a comparison of scales and exploration of treatment efficacy outcome measures. Acad Emerg Med. 2015;22(6):685-93. doi: https://doi. org/10.1111/acem.12685

17. Nunnally JC, Bernstein IH. Psychometric theory. 3rd ed. New York: McGraw Hill; 1994. (McGraw Hill series in psychology).

18. Hair JF, Black WC, Babin BJ, et al. Análise multivariada de dados. 6. ed. Porto Alegre, SC: Bookman; 2009.

19. Dancey CP, Reidy J. Estatística sem matemática: para psicologia usando SPSS para Windows. 3. ed. Porto Alegre, SC: Artmed; 2006.

20. Pereira TJ, Puggina AC. Validation of the self-assessment of communication skills and professionalism for nurses. Rev Bras Enferm. 2017;70(3):588-94. doi: https://doi. org/10.1590/0034-7167-2016-0133

21. Escobar Y, Cajaraville G, Virizuela JA, et al. Incidence of chemotherapy-induced nausea and vomiting with moderately emetogenic chemotherapy: ADVICE (Actual Data of Vomiting Incidence by Chemotherapy Evaluation) study. Support Care Cancer. 2015;23(9):2833-40. doi: https://doi.org/10.1007/ s00520-015-2809-3. Erratum in: Support Care Cancer. 2015;23(9):2841. doi: https://doi.org/10.1007/ s00520-015-2842-2

22. Pearce A, Hass M, Viney R, et al. Incidence and severity of self-reported chemotherapy side effects in routine care: a prospective cohort study. PLoS One. 2017;12(10):e0184360. doi: https://doi.org/10.1371/ journal.pone. 0184360

23. Puri S, Hyland KA, Weiss KC, et al. Prediction of chemotherapy-induced nausea and vomiting from patient-reported and genetic risk factors. Support Care Cancer. 2018;26(8):2911-8. doi: https://doi. org/10.1007/s00520-018-4120-6

24. Razvi Y, Chan S, McFarlane E, et al. ASCO, NCCN, MASCC/ESMO: a comparison of antiemetic guidelines for the treatment of chemotherapy-induced nausea and vomiting in adult patients. Support Care Cancer. 2019;27(1):87-95. doi: https://doi.org/10.1007/s00520018-4464-y

25. Adel N. Overview of chemotherapy-induced nausea and vomiting and evidence-based therapies. Am J Manag Care. 2017;23(14 Suppl):S259-S65. 
26. Bošnjak SM, Gralla RJ, Schwartzberg L. Prevention of chemotherapy-induced nausea: the role of neurokinin-1 $\left(\mathrm{NK}_{1}\right)$ receptor antagonists. Support Care Cancer. 2017;25(5):1661-71. doi: https://doi.org/10.1007/ s00520-017-3585-z

27. Morrow GR. Methodology and assessment in clinical anti-emetic research: a meta-analysis of outcome parameters. Br J Cancer Suppl. 1992;19:S38-S41.

28. Maia AES, Grello FAC, Cunha KC. Perfil sociodemográfico e clínico de pacientes com câncer cadastrados no programa de visita domiciliar de um hospital da rede pública. Rev Bras Cancerol. 2021;67(2): e-05864. doi: https://doi.org/10.32635/2176-9745. RBC.2021v67n2.864

29. Roscoe JA, Morrow GR, Aapro MS, et al. Anticipatory nausea and vomiting. Support Care Cancer. 2011;19(10):1533-8. doi: https://doi.org/10.1007/ s00520-010-0980-0

30. Castro MC, Araújo SA, Mendes TR, et al. Effectiveness of antiemetics in control of antineoplastic chemotherapyinduced emesis at home. Acta Paul Enferm. 2014;27(5):412-18. doi: https://doi.org/10.1590/19820194201400069 